\title{
Prospective study of the feasibility of point-of-care testing strategy for carbapenem-resistant organism detection
}

\section{(ㄷ)(1) $\odot$}

\author{
Authors \\ Institutions \\ 1 Division of Gastroenterology and Hepatology, Mayo \\ Clinic, Scottsdale, AZ, USA \\ 2 Division of Laboratory Medicine, Mayo Clinic, \\ Scottsdale, AZ, USA \\ 3 Cepheid, Sunnyvale, CA, USA
}

Rahul Pannala ${ }^{1}$, Bruce Baldwin ${ }^{1}$, Vijay Aluru ${ }^{1}$, Thomas E. Grys ${ }^{2}$, Jordan Holmes ${ }^{1}$, Laurence J. Miller ${ }^{1}$, M. Edwyn Harrison ${ }^{1}$, Cuong C. Nguyen ${ }^{1}$, Fred C. Tenover ${ }^{3}$, David Persing ${ }^{3}$, Douglas O. Faigel ${ }^{1}$

submitted 25.1.2017

accepted after revision $\quad 10.10 .2017$

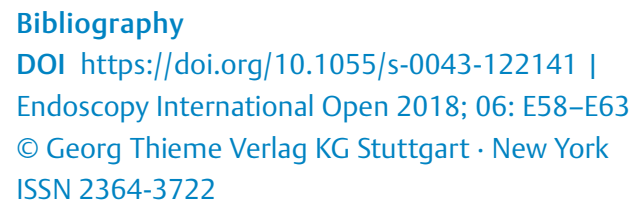

Corresponding author

Rahul Pannala, MD, MPH, Mayo Clinic, 13400 E Shea Blvd, Scottsdale, AZ 85259, USA

Fax: +1-480-301-6737

Pannala.Rahul@mayo.edu

\section{ABSTRACT}

Background/aims In an investigator-initiated, prospective study, we evaluated the feasibility of a five-gene sequence point-of-care (POC) testing strategy (Xpert CARBA-R Assay, Cepheid Inc., Sunnyvale, CA, USA), compared to reference laboratory PCR (48-72 hours turnaround time, two gene sequences), in patients undergoing endoscopic retrograde cholangiopancreatography (ERCP) and in a hospital outbreak investigation.

Methods After informed consent, patients undergoing ERCP (September 2015 - April 2016, $n=191$ ) at Mayo Clinic and potential hospital contacts $(n=9)$ of an index carbapenem-resistant organism (CRO)-positive inpatient were included. Two rectal swabs, one each for reference and POC assays were obtained. The Xpert CARBA-R Assay enables qualitative rapid detection of five beta-lactamase gene sequences associated with carbapenem-non-susceptibility in Gram-negative bacteria. Feasibility parameters (specimen processing and assay run time, ease of use) and percent agreement between the tests were calculated using JMP Pro11 (SAS Corp, Cary, NC, USA).

Results Mean age was $62 \pm 15$ years; 108 (54\%) were male. Both tests were successfully performed in all patients. The POC test was rated by endoscopy nurses as easy/very easy to conduct in 193 patients (97\%); median assay run time and median time for specimen collection and processing were 55 minutes (interquartile range IQR: 53 - 55 minutes) and 3 minutes (IQR: 3 - 6 minutes), respectively. In 200/201 (99.5\%) tests, there was agreement between the POC and reference PCR.

Conclusions The more comprehensive POC CRO testing of patients in the endoscopy suite is feasible and results are available in $<1$ hour. This strategy may enable rapid risk stratification of duodenoscope exposure to CRO and potentially improve operational efficiency and decrease costs.

\section{Introduction}

Carbapenem-resistant organisms (CRO) are defined as Gramnegative bacterial isolates that are resistant to any carbapenem or are documented to produce a carbapenemase [1]. Typical CRO pathogens include members of the Enterobacteriaceae (carbapenem-resistant Enterobacteriaceae, CRE), as well as Pseudomonas and Acinetobacter species. Nosocomial transmission of CRO is a serious public health concern and invasive infections are associated with a very high mortality rate. Treatment options for CRO are limited, as these bacteria have been reported to be resistant to several other antimicrobial agents in addition to carbapenems.

In the United States, the earliest CRO infections were related to bacteria that produce the Klebsiella pneumoniae carbapenemase (KPC) [2]. Subsequently, several other carbapenemases have been identified including New Delhi metallo $\beta$-lactamase (NDM), Verona Integron-encoded metallo- $\beta$-lactamase (VIM), Oxacillinase-48-type carbapenemase (OXA-48), and Imipenemase metallo- $\beta$-lactamase (IMP). While most infections in the United States carry the KPC and NDM resistance genes, more 
recently, there have been outbreaks caused by VIM and OXA-48 producing bacteria $[3,4]$.

Duodenoscope-associated nosocomial transmission of carbapenem-resistant Enterobacteriaceae (CRE) among patients undergoing endoscopic retrograde cholangiopancreatography (ERCP) has been reported in several healthcare facilities in the United States and Europe [5-9]. Furthermore, an outbreak of VIM-2-producing Pseudomonas aeruginosa (non-Enterobacteriaceae species) was reported in Europe that was linked to the design of the recess under the elevator of the duodenoscope [9]. These reports have led to widespread concern with regard to duodenoscope-associated CRO infections.

The US Food and Drug Administration (FDA) noted that the complex design of the duodenoscope may impede effective reprocessing and that meticulous cleaning before high-level disinfection will reduce but may not entirely eliminate the risk of transmitting infection [10]. In this regard, the FDA recommends adherence to the multisociety guidelines on flexible endoscope reprocessing to minimize the chances of transmission. However, CRO transmission through infected duodenoscopes has been reported despite adherence to manufacturerrecommended high-level disinfection protocol [7]. Several patient-related factors including the placement of a biliary stent and inpatient status have been identified as independent risk factors for CRO transmission [11]. While an optimal prevention or surveillance strategy has not been developed, double cycles of washing and high-level disinfection, patient screening using culture or polymerase chain reaction (PCR) testing on rectal swabs, endoscope culturing for intermittent surveillance, or low temperature sterilization using ethylene oxide (EtO) have been suggested as additional measures, depending on local availability of resources and expertise [12].

Reference laboratory-based PCR tests for patient surveillance are not widely available and usually require 48-72 hours turnaround time for result availability. In addition, the reference laboratory PCR tests available at most institutions are designed to detect only KPC and NDM carbapenemase gene sequences. A multiplex PCR test that identifies a broad array of beta-lactamase families will allow for a more robust surveillance and diagnostic approach.

Duodenoscope surveillance with routine cultures is cumbersome, labor intensive, and of uncertain value. In addition, although duodenoscope sampling methods have been proposed, the sensitivity or specificity of these approaches has not been characterized. EtO sterilization is effective when duodenoscopes are adequately free of biological debris, but is expensive and there have been reports of endoscope damage with this technique [13]. In addition, as duodenoscopes are quarantined during culture or sterilization, these strategies usually require acquisition of additional endoscopes. In a study of the cost utility of culture and hold strategy or ERCP and EtO sterilization, both strategies were noted to be cost prohibitive given the low prevalence of CRO at most institutions [14]. In particular, this analysis noted that for the EtO sterilization strategy to be costeffective, the prevalence of CRE should exceed $24 \%$ or that the cost of EtO should be less than $\$ 84$ per ERCP, both of which are currently unrealistic scenarios [14]. Therefore, there is an un- met need to rapidly evaluate the CRO status of patients undergoing ERCP, ideally using a simple, more comprehensive, pointof-care test in the endoscopy suite, such that only duodenoscopes exposed to CRO patients can be targeted for sterilization/culture methods.

We hypothesized that a rapid, more comprehensive POC CRO testing strategy using the Xpert Carba-R assay (Cepheid, Sunnyvale, California, United States) is feasible in a high throughput endoscopy suite, compared to the current reference laboratory PCR. While the successful use of a similar assay for methicillin-resistant Staphylococcus aureus (MRSA) (Xpert MRSA, Cepheid) in a POC setting in the orthopedic and vascular ward [15] and Clostridium difficile assay (Cepheid GeneXpert) in the intensive care unit and geriatric wards [16] has previously been reported, a similar use in the endoscopy suite has not been evaluated. In an investigator-initiated, prospective study of patients undergoing ERCP, and testing of potential contacts of an index CRO-positive inpatient as a part of hospital outbreak investigation, we evaluated the feasibility of the POC CRO testing strategy, compared to the reference laboratory PCR.

\section{Methods}

The Mayo Clinic institutional review board approved the study. All consecutive patients undergoing ERCP from September 2015 to April 2016 and who gave informed consent were included in the study $(n=191)$. In addition, as a part of a concurrent hospital outbreak investigation, potential hospital contacts $(n=9)$ of an index hospital inpatient with CRO were also included in the study. Therefore, a total of 201 patients were included in the study.

POC testing was performed using a Research Use Only version of the Xpert CARBA-R assay (Cepheid), which is a qualitative, in vitro automated real-time PCR-based assay. The assay has since been FDA-cleared for testing both carbapenem nonsusceptible bacterial isolates and rectal swab samples. The Xpert CARBA-R test enables rapid detection of five common gene sequences (those encoding KPC, NDM, VIM, OXA-48, and IMP) contributing to carbapenem non-susceptibility. The reported performance characteristics of the assay for these gene sequences when performed on rectal swabs range in sensitivity from $94.5 \%$ to $100 \%$ and in specificity from $99.1 \%$ to $99.7 \%$ [17]. It is a closed cartridge-based system that is part of a wellestablished platform (GeneXpert system) that is widely utilized for similar assays for methicillin-resistant Staphylococcus aureus (MRSA), Influenza/Respiratory Syncytial Virus, and Clostridium difficile testing. The system consists of a GeneXpert instrument (GX), personal computer, and disposable fluidic cartridges that are designed to complete sample preparation and real-time PCR for detection of CRO in about 50 minutes. Each instrument contains from 1 to 80 modules that are each capable of independently performing the real-time PCR tests, enabling a continuous workflow. The module contains a syringe drive for dispensing fluids, ultrasonic horn for lysing cells or spores, and an I-CORE thermocycler for performing real-time PCR and target detection. The GX is connected to a personal computer that allows the operator to run a test using a simple 


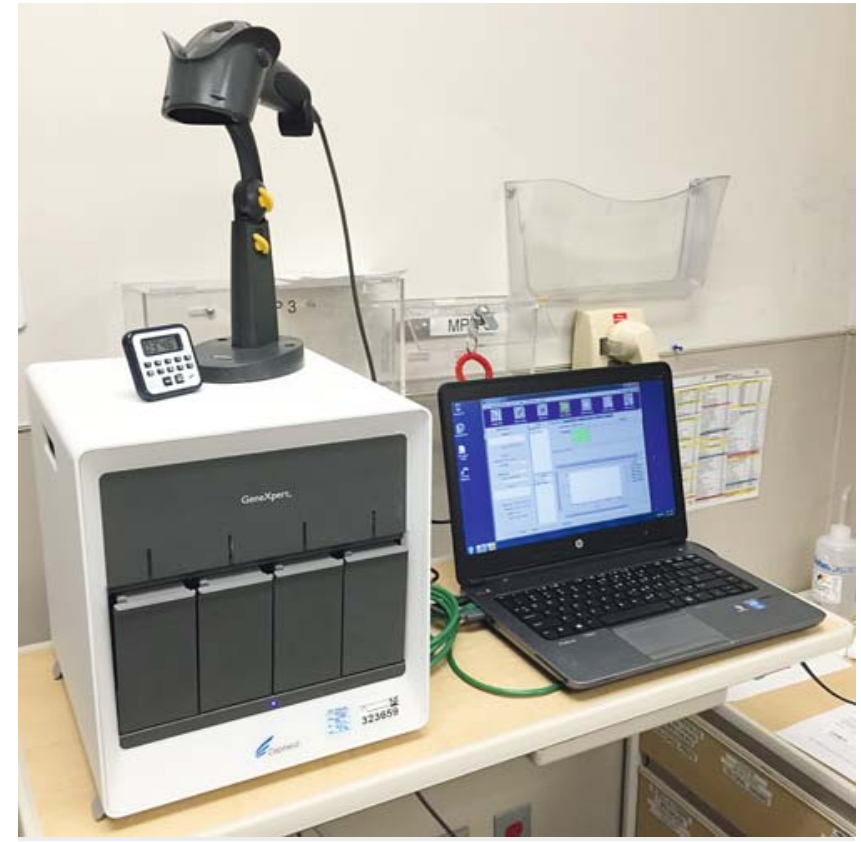

- Fig. 1 Four-module Xpert instrument that runs the CARBA-R CRO (Cepheid, Sunnyvale, California, United States) assay with associated laptop computer in the preoperative area of the endoscopy suite.

user interface. For the purposes of POC testing, the assay system and the connected laptop computer were placed in the preoperative area of the endoscopy suite ( $>$ Fig. 1$)$. A verification study demonstrating expected performance of the POC PCR assay was successfully performed by the Mayo Hospital Microbiology Department, who underwent training by the vendor at the same time as endoscopy nursing staff.

For patients undergoing ERCP, our institutional protocol is to obtain a rectal swab in the preoperative area or the procedure room for reference laboratory PCR testing. Duodenoscopes are high-level disinfected twice and sequestered until assay results are available. If the assay is positive, the duodenoscope is then sterilized using EtO. For patients included in the study, an additional rectal swab was obtained for POC testing. All patients were tested. As with the reference laboratory PCR test, the POC test was considered valid for 90 days and if the patient had a repeat ERCP within that duration, the test was not repeated nor were patients enrolled again into the study. The endoscopy nurses performed the initial set-up of the POC assay in the endoscopy suite with the assistance of the microbiology department. Trained endoscopy nurses also performed the collection of all rectal swabs, and also performed the POC PCR testing on all of the samples. For the POC assay, the rectal swabs were placed in a vial containing a sample reagent, the specimen was vortexed for 10 seconds, then transferred to the Xpert CARBA-R cartridge using the disposable pipette provided with the test kit, and the GeneXpert instrument was then run.

The reference PCR that is utilized by our institution is a laboratory-developed test that utilizes a multiplex real-time PCR
- Table 1 Demographic and clinical characteristics of patients included in the study $(n=201)$.

\begin{tabular}{|l|c|}
\hline Parameter & \\
\hline Test indication, $\mathrm{n}(\%)$ & $191(95)$ \\
\hline - ERCP & $10(5)$ \\
\hline - Hospital investigation & $62 \pm 15$ \\
\hline Age, mean \pm SD, $\mathrm{y}$ & $108(54)$ \\
\hline Males, $\mathrm{n}(\%)$ & $69(35)$ \\
\hline Previous ERCP, $\mathrm{n}(\%)$ & $82(41)$ \\
\hline Invasive malignancy history, $\mathrm{n}(\%)$ & $28(14)$ \\
\hline Pancreatobiliary malignancy, $\mathrm{n}(\%)$ & $57(29)$ \\
\hline Recent antibiotic use, $\mathrm{n}(\%)$ & $11(6)$ \\
\hline Urinary tract infections, $\mathrm{n}(\%)$ & $1(0.5)$ \\
\hline Skilled nursing facility, $\mathrm{n}(\%)$ & $131(66)$ \\
\hline Previous endoscopy, $\mathrm{n}(\%)$ & $40(20)$ \\
\hline Diabetes mellitus, $\mathrm{n}(\%)$ & \\
\hline ERCP, endoscopic retrograde cholangiopancreatography. & \\
\hline
\end{tabular}

approach. The assay targets KPC and NDM gene sequences and has been validated for stool or rectal swabs [18].

Patient demographic and clinical data were abstracted from the electronic medical record (EMR). Reference PCR results were also abstracted from the EMR when they were reported, usually after $48-72$ hours. For assessment of the feasibility of the POC assay, the endoscopy nurse who performed the rectal swab documented the time required for specimen collection and processing, which was calculated as the time from obtaining the rectal swab to the start of the assay, the ease of use in terms of specimen collection and handling, and running the assay recorded on a five-point Likert scale (1: challenging, 5: very easy), and the assay run time (time to result availability from the start of the assay). Percent agreement between the POC assay and the reference PCR was calculated. Positive results, either on the POC assay or the reference PCR, were confirmed by culture. Statistical analyses were performed using JMP Pro 11 (SAS Corporation, Cary, North Carolina, United States).

\section{Results}

Of the 201 patients included in the study, 191 (95\%) were those who underwent ERCP; 10 (5\%) included an index-hospitalized patient with CRO and nine potential contacts who were patients in the same hospital pod. Mean age was $62 \pm 15$ years; 108 (54\%) were males ( Table 1 ). Of the patient-related risk factors that may predispose to CRO infection, $82(41 \%)$ had a current or previous history of invasive malignancy; 28 (14\%) had pancreatobiliary malignancy. Recent antibiotic use (within 30 days of the procedure) was noted in 57 (29\%). A recent (<30 days of the procedure) urinary tract infection was reported in $11(6 \%)$. 
Table 2 Feasibility parameters of the point-of-care (POC) assay (Xpert CARBA-R CRO assay, Cepheid, Sunnyvale, CA, United States).

\begin{tabular}{|c|c|}
\hline \multicolumn{2}{|l|}{ Assay characteristics $(n=201)$} \\
\hline $\begin{array}{l}\text { Specimen collection and handling time, median } \\
\text { (IQR), min }\end{array}$ & $3(3-6)$ \\
\hline \multicolumn{2}{|l|}{ Ease of use ${ }^{1}$} \\
\hline - Very easy, n (\%) & $107(54)$ \\
\hline - Easy, n (\%) & $86(43)$ \\
\hline Run time, median (IQR), min & $55(53-55)$ \\
\hline
\end{tabular}

Among patients undergoing ERCP, CRO was detected in one patient $(0.5 \%)$ and this was detected by both assays and confirmed with culture. The index hospitalized CRO-positive patient was confirmed to be positive with both assays and culture. None of the hospital contacts tested positive for CRO. A concordant result between the POC assay and the reference PCR was noted in 200 patients (percent agreement: 99.5\%). One patient tested positive for the VIM target by the POC assay, which was not assessed by the reference PCR. The sample was cultured and confirmed to contain a VIM-positive, multidrug-resistant P. aeruginosa (i.e. a non-Enterobacteriaceae).

A median time of 3 minutes (interquartile range 3-6 minutes) was reported for specimen collection and processing for the POC assay ( $\triangleright$ Table 2 ). The median assay run time was 55 minutes (IQR 53-55 minutes) and endoscopy nurses who performed the assays rated the process as easy or very easy in 193 assays (97\%).

\section{Discussion}

In a prospective, investigator-initiated study of 201 patients, which included 191 patients undergoing ERCP and 10 patients who were part of a hospital CRO outbreak evaluation, we report that the prevalence of CRO infection is very low $(0.5 \%)$ in patients undergoing ERCP at a tertiary care US hospital. Preprocedure POC testing for CRO with the Xpert CARBA-R CRO assay is feasible with a turnaround time of $<1$ hour. This strategy enables rapid assessment of patient's CRO status and immediate risk stratification of duodenoscope exposure to CRO. In addition, the POC assay is easy to use by endoscopy nursing staff, and has a $99.5 \%$ agreement with the current reference laboratory PCR assay. Furthermore, we also report the detection of VIM-positive P. aeruginosa (non-Enterobacteriaceae) in one patient; this was detected by the POC test but not by the reference assay, which only tested for KPC and NDM. The Xpert CAR$B A-R$ test detects the five classes of carbapenemase genes regardless of the bacterial species, allowing detection of not only Enterobacteriaceae, but also Pseudomonas and Acinetobacter species that are known to harbor these genes. This is the first report of detection of VIM-positive P. aeruginosa strain in patients undergoing ERCP in the United States.
There are limited data on the prevalence of CRO among patients undergoing ERCP. In a retrospective analysis of patients undergoing ERCP from 2012 to 2013 during which 1149 ERCPs were performed, Ross et al. identified 32 patients with multidrug-resistant Escherichia coli [7]. Other studies have only reported the prevalence of infections in patients exposed to contaminated duodenoscopes $[5,8,13]$. In our study, all consecutive patients undergoing ERCP in the study duration were included and our results indicate that, even in a tertiary care center, the prevalence of CRO infections is low. CRO detection and surveillance strategies would need to factor in the low prevalence to be cost-effective. In an analysis of various strategies, Almario et al. reported that ERCP with FDA-recommended endoscope reprocessing was the most cost-effective strategy; culture and hold, and EtO sterilization were noted to be cost-prohibitive [14]. The authors also reported that for the culture and hold strategy to be cost-effective, the pre-test probability of CRO should exceed $24 \%$, which is currently an unrealistic scenario.

Our aim was to evaluate a POC strategy for rapid assessment of CRO status of patients undergoing ERCP such that this information could enable risk stratification of duodenoscope exposure at the time of the procedure. This is the first study to demonstrate feasibility of this approach; endoscopy nurses performed all testing and most rated the process as easy/very easy. These data indicate that this approach is generalizable and this strategy may be particularly useful for two reasons. One is to implement infectious precautions for patients carrying CRO to minimize spread (similar to screening for MRSA or vancomycin-resistant enterococci [VRE]). The other is to triage reprocessing of endoscopes to minimize the need for EtO and decrease the need to sequester duodenoscopes for a longer period and, consequently, the need for endoscopy units to purchase additional duodenoscopes. Furthermore, given the concerns of endoscope damage with EtO sterilization [13] and the lack of long-term data on endoscope durability with repeated chemical sterilization, POC testing-based utilization of EtO sterilization may help mitigate these risks. In addition, for practices that do not routinely use a reference laboratory service, the POC strategy allows ready access to information that would otherwise not be available. Further studies in other practice settings, including institutions with high CRO prevalence, are needed to validate our results. To our knowledge, our study is the first report of a POC assay being utilized for screening patients. Other institutions have reported on utilizing laboratorybased PCR tests to screen patients in combination with other strategies [19].

We also demonstrated in a small subset of hospitalized patients that this assay can be useful for hospital outbreak investigations with results available within 1 hour. This would also potentially allow for rapid implementation of necessary infection control precautions.

This study was not designed to evaluate the cost-effectiveness of this POC testing strategy. Future studies should comprehensively evaluate the cost effectiveness of this approach, addressing issues such as cost of culture and hold, universal EtO sterilization, avoidance of undetected spread, and the 
need for additional duodenoscopes. However, a simple comparison of the potential cost of this strategy relative to others may help estimate the potential cost savings. Assuming a universal EtO sterilization strategy and utilizing the composite cost estimate for EtO (including the cost of new duodenoscopes, FDA-recommended reprocessing, sterilization, and the cost of replacing duodenoscopes damaged by EtO) published in the literature (\$1044) [14], the cost for sterilizing duodenoscopes utilized in ERCP patients included in the study $(n=191)$ would be $\$ 199404$. Assay costs would be approximately $\$ 10505$ (\$55 per assay). In this study population, the use of a POC assay and the subsequent need to only sterilize two duodenoscopes (\$2088) would lead to a projected saving of $\$ 186511$.

Given the low prevalence of CRO among patients undergoing ERCP in our study, we were not able to evaluate patient and disease characteristics, which may predispose to CRO colonization and consequent duodenoscope exposure. Reported risk factors in other studies include biliary stent placement, a diagnosis of cholangiocarcinoma, and inpatient status [11]. We tested all patients undergoing ERCP and considered the test result valid for a duration of 3 months. The impact of patient or technical issues that would influence the yield of CRE on the POC assay was not tested. However, patients undergoing ERCP do not utilize a bowel preparation or other medications that may impact the test. Rectal swab testing in the outpatient setting may be ideal and may better represent routine flora but the procedure is often performed in patients hospitalized with cholangitis or other pancreatobiliary diseases and therefore this strategy is not feasible in all patients. As the reported performance characteristics of the POC assay, when performed on rectal swabs, range in sensitivity from $94.5 \%$ to $100 \%$, and in specificity from $99.1 \%$ to $99.7 \%$ [17], in our opinion, patient characteristics are unlikely to have a substantial impact on the overall yield.

The effectiveness of duodenoscope surveillance utilizing this assay was also not evaluated in the current study. An optimal strategy for surveillance of duodenoscopes has not been established. Microbiological culture of duodenoscopes and adenosine triphosphate (ATP) quantification methods have been proposed but these methods have limitations and are not utilized widely [20-22]. PCR-based assays, when utilized for endoscope testing, also have similar limitations and do not have the capability of distinguishing live from dead organisms. In a randomized study of three strategies for endoscope reprocessing where culture from the duodenoscopes was performed, bacterial growth of greater than zero colony-forming units (cfu) was noted in 16-23\% of all cultures [21] including with EtO sterilization. PCR-based assays are likely to be more sensitive and therefore may have limited clinical applicability to routine clinical surveillance of duodenoscopes.

Another important finding in our study is the detection of VIM-positive P. aeruginosa, a multidrug-resistant organism (MDRO) that is not an Enterobacteriaceae. VIM and OXA-48 producing CRO outbreaks have recently been reported in the United States $[1,4]$. The POC assay that was used in the present study tests for five carbapenemase gene sequences, including
VIM and OXA-48. In contrast, the current reference PCR is designed to test for KPC and NDM sequences only. This highlights the need to expand testing to detect CRO that are non-Enterobacteriaceae, since CRO of any species could be transmitted through contaminated endoscopes.

In conclusion, we report that CRO prevalence is low in patients undergoing ERCP in our center, and that implementation of a POC PCR-based testing strategy that detects all five carbapenemase gene sequences is feasible in a busy endoscopy unit. In every case, the results were available in less than 1 hour, and the results were accurate in all cases in detecting the KPC and NDM targets detected by the current reference laboratory PCR assay. Furthermore, the POC assay detected a VIM-positive MDRO (non-Enterobacteriaceae) that was not detected by the reference PCR assay. Endoscopy nurses performed the testing and rated the process as easy/very easy in nearly all cases. A POC testing strategy has the potential to enable rapid assessment of the CRO status of patients undergoing ERCP and immediate risk stratification of duodenoscope exposure.

\section{Acknowledgements}

American Society of Gastrointestinal Endoscopy Duodenoscope Infection Control Research Award, 2015.

\section{Competing interests}

Drs. Tenover and Persing are employees of Cepheid Inc. Other authors have no relevant disclosures.

\section{References}

[1] Centers for Disease Control and Prevention. Facility guidance for carbapenem-resistant enterobacteriaceae (CRE) - November 2015 update - CRE toolkit. Atlanta: GA; 2015

[2] Yigit H, Queenan AM, Anderson G] et al. Novel carbapenem-hydrolyzing beta-lactamase, KPC-1, from a carbapenem-resistant strain of Klebsiella pneumoniae. Antimicrob Agents Chemother 2001; 45: $1151-1161$

[3] Centers for Disease Control and Prevention (CDC). Update: detection of a verona integron-encoded metallo-beta-lactamase in Klebsiella pneumoniae - United States, 2010. MMWR Morb Mortal Wkly Rep 2010; 59: 1212

[4] Lyman M, Walters M, Lonsway D et al. Notes from the field: carbapenem-resistant Enterobacteriaceae producing OXA-48-like carbapenemases - United States, 2010-2015. MMWR Morb Mortal Wkly Rep 2015; 64: 1315-1316

[5] Epstein L, Hunter JC, Arwady MA et al. New Delhi metallo-beta-lactamase-producing carbapenem-resistant Escherichia coli associated with exposure to duodenoscopes. Jama 2014; 312: 1447-1455

[6] Centers forDisease Control and Prevention. Notes from the Field: New Delhi metallo-beta-lactamase-producing Escherichia coli associated with endoscopic retrograde cholangiopancreatography - Illinois, 2013. MMWR Morb Mortal Wkly Rep 2014; 62: 1051

[7] Ross AS, Baliga C, Verma P et al. A quarantine process for the resolution of duodenoscope-associated transmission of multidrug-resistant Escherichia coli. Gastrointest Endosc 2015; 82: 477-483 
[8] Wendorf KA, Kay M, Baliga C et al. Endoscopic retrograde cholangiopancreatography-associated AmpC Escherichia coli outbreak. Infect Control Hosp Epidemiol 2015; 36: 634-642

[9] Verfaillie C], Bruno M], Voor in 't Holt AF et al. Withdrawal of a noveldesign duodenoscope ends outbreak of a VIM-2-producing Pseudomonas aeruginosa. Endoscopy 2015; 47: $493-502$

[10] FDA. Design of endoscopic retrograde cholangiopancreatography (ERCP) duodenoscopes may impede effective cleaning: FDA Safety Communication. 2015

[11] Kim S, Russell D, Mohamadnejad M et al. Risk factors associated with the transmission of carbapenem-resistant Enterobacteriaceae via contaminated duodenoscopes. Gastrointest Endosc 2016; 83: 1121 1129

[12] ASGE. Transmission of CRE bacteria through endoscopic retrograde cholangiopancreatography (ERCP) - interim guidance. ASGE; 17.03 2015

[13] Naryzhny I, Silas D, Chi K. Impact of ethylene oxide gas sterilization of duodenoscopes after a carbapenem-resistant Enterobacteriaceae outbreak. Gastrointest Endosc 2016; 84: 259-262

[14] Almario CV, May FP, Shaheen NJ et al. Cost utility of competing strategies to prevent endoscopic transmission of carbapenem-resistant enterobacteriaceae. Am J Gastroenterol 2015; 110: 1666 - 1674

[15] Parcell B], Phillips G. Use of Xpert(R) MRSA PCR point-of-care testing beyond the laboratory. J Hosp Infect 2014; 87: 119-121
[16] Goldenberg SD, Bisnauthsing KN, Patel A et al. Point-of-care testing for clostridium difficile infection: a real-world feasibility study of a rapid molecular test in two hospital settings. Infect Dis Ther 2014; 3 : 295-306

[17] Cepheid. Sunnyvale, CA, USA: Cepheid; http://cepheid.com/ administrator/components/com_productcatalog/library-files/ 93eee8a71f1 ef27462a70a0ba22fd8f4-a592a437b2387d232128ea7dda751100-Xpert-Carba-R-Datasheet-US-0561-02.pdf

[18] Vasoo S, Cunningham SA, Kohner PC et al. Rapid and direct real-time detection of blaKPC and blaNDM from surveillance samples. J Clin Microbiol 2013; 51: 3609-3615

[19] Smith ZL, Dua A, Saeian K et al. A novel protocol obviates endoscope sampling for carbapenem-resistant Enterobacteriaceae: experience of a center with a prior outbreak. Dig Dis Sci 2017; 62: 3100 - 3109

[20] Sethi S, Huang RJ, Barakat MT et al. Adenosine triphosphate bioluminescence for bacteriologic surveillance and reprocessing strategies for minimizing risk of infection transmission by duodenoscopes. Gastrointest Endosc 2017; 85: 1180-1187 e1

[21] Snyder GM, Wright SB, Smithey A et al. Randomized comparison of 3 high-level disinfection and sterilization procedures for duodenoscopes. Gastroenterology 2017; 153: $1018-1025$

[22] Olafsdottir LB, Wright SB, Smithey A et al. Adenosine triphosphate quantification correlates poorly with microbial contamination of duodenoscopes. Infect Control Hosp Epidemiol 2017; 38: 678-684 